. 


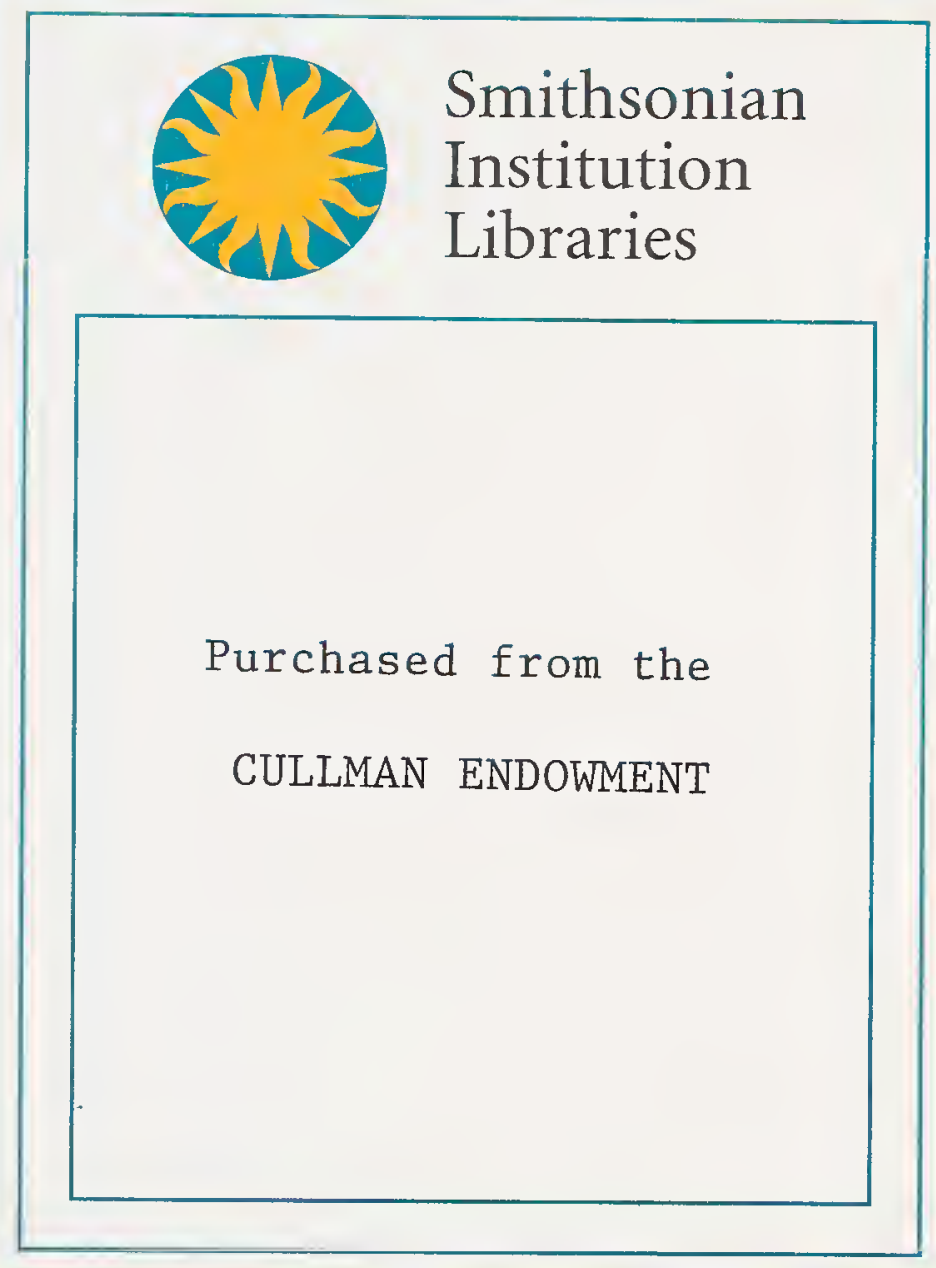




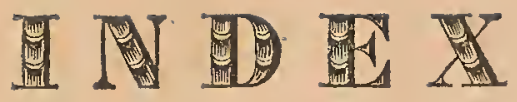

\section{PLANTARUM SUCCULENTARUM}

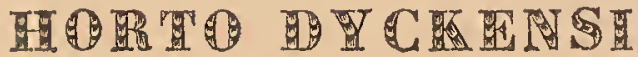

G U L T A R U M.

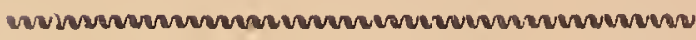

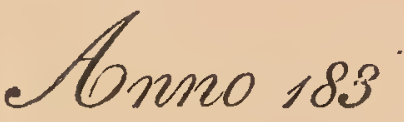

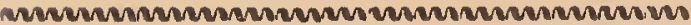

AQUISGRANI,

Typis J. J. B E A $\forall$ F 0 R.

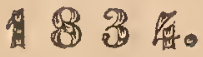





\section{PLANT压 SUCCULENTE}

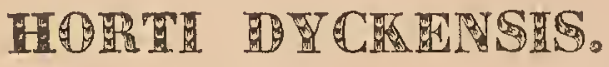

Agave Lin. (Cavtescentes.)

- angustifolia Haw.

- cantala Haw.

- Commelini Nob.

- foetida Lin. Furcora giganlea Vent.

- lurida Ait.

- tuberosa Ait.

\section{- \\ $-$}

(ACAULES.)

Nonnullo forsan Caulescentes.

- americana Lin.

- fol. viridibus, luteo-marginatis.

- - fol. luteis, viridi-marginatis.

- fol. obsolete striatis.

- - fol. viridibus, luteo-striatis.

- - fol. luteis, viridi-striatis.

- atrovirens Karw.

- attenuata H. Berol.

- bromeliafolia H. Dyck. 
- bulbifera $H$. Dyck.

- flavescens H. Monac.

- geminiflora Brand. (Litra A Tagli.)

- heteracantha Zuccar.

- Ixtli Karw.

- Karatto Mill.

- laxa H. Monac.

- macroacantha Zuccar.

- madagascariensis Haw.

- Milleri Haw. virginica Mill.

- polyanthoides Schied?

- potatorum Zuccar.

- pugioniformis Zuccar.

- pulchra H. Monac.

- punctata H. Berol.

- rubescens Nob.

- Scolymus Karw.

- sobolifera Herm. vivipara Hort.

- striata Zuccar. (Bonapantea juncea Hort.)

- univittata Haw.

- veracrucis Mill.

- vivipara Lin. polygona Comm.

- yuccæfolia Red. Lil. 
(HERBACKAE.)

- brachystachys Cav.

- spicala Cav.

- virginica Jacq.

Arzoon canariense Lin。

- glinoides Lin.

- hispanicum Lin.

- rigidum Lin.

Aroe Lin.

(Apicrae Willd.)

(IMBRICATAE.)

- imbricata Haw.

- pentagona stricta Hcuv.

- - torta Haw.

- quinguangularis Schult.

- spiralis Hazv.

- spirella Haw.

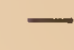

\section{(FOLIOLOSAE.)}

- aspera Haw.

- foliolosa Haw. 


\section{(6) ,}

HAWORTHIAE Haw.

(TRIQUETRAE.)

- asperiuscula Haw.

- concinna Haw.

- cordifolia IIaw.

- indurata Haw.

- subtortuosa Schult. pseudo-iortuosa Nob.

- corquata Haw. pseudo-tortuosa elongata Nob..

- viscosa major Haw.

- minor Haw.

\section{(TORTUOSAE.)}

- Irybrida Nob.

- - asperior Nob.

- rigida major $D C$. expansa Haw.

- minor Nob. patula WT.

- subrigida Schult. pseudo-rigida Nob.

- tortuosa Haw. curta Haw.

- major Nob.

(ALBLCANTES.)

- albicans Haw. loevis Haw. marginata Lamk.

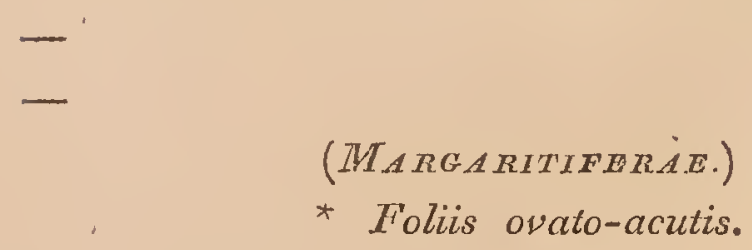

- erecta Haw. margar. major $I V$.

- - minor NoZ. H. minor Haw.

- latevirens Nob. 
- granata $W$.

- - minor Nob. Ax. margaritif, DC.

- margaritifera Nob. Haw?

- papillosa Nob.

- minor Nob.

- semiglabrata Haw.

- semimargaritifera maxima Nob.

- major Nob.

- - glabrata Nob.

- - multipapillosa Nob. multiperlata Haw.

\section{$-$}

** Foliis cuspidato-attenuatis. $\dagger$ Utrinque tuberculatis.

\section{- attenuata Haw.}

- - clariperla Nob. H. clariperla Haw.

- Radula Jacq.

- rugosa Nob. Radula asperior Haw.

- perviridis Nob.

- subattenuata Nob. Radula magniperl. Haw.

- subulata Nob. Radula loevior Haw.

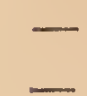

\footnotetext{
it Supra lavibus.

- coarctata Haw.

- fasciata $T$.

- major Nob.

- glabrata $N a b$.
} 
- Reinwardi Nob.

- subfasciata Nob. fasciata major Haw.

- recurva Haw.

(LURIDAE.)

- - loevior H. Dick.

- scabra Haw.

- sordida Haw.

\section{(TESSELLATAE)}

- parva Haw.

- tessellata Haw.

$-$

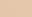

$(R E T U S A E$.

- asperula Haw.

- mirabilis Haw.

- multifaria Haw.

- mutica Haw.

- retusa Lin.

- turgida Haw.

$($ RETICULATAE)

- atrovirens $D C$.

- lætevirens Haw.

- reticulata Haw. Pumilio Jacq. herbacea DC. 
(EIMPIDAE.)

- altilinea How.

- cuspidara Haw.

- cymbæfolia Haw. concava Haw.

- - fol. erectioribus Haw.

- planifolia Haw.

- arachnoides Mill.

(SETOSAE.)

- Klugii H. Berol.

- setosa Haw.

- translucens Haw.

$-$

$-$

(LORATAE.)

- chloracantha Haw.

- stenophylla Schult. angustifolia Haw.

$-$

(BOWIEAE.)

- Bowiea Schult. - Bowiea africana $H a$

\footnotetext{
ALOAE. Haw.

(ECHINATAE.)

- acuminata Haw.

- - minor Nob.

- - spinosior Nob.
} 
- humilis Lam.

- Latifolia Haw.

- incurva Haw. humilis Jacq.

- - glaucior Nob.

- longiaristata Schult, aristata Haw.

- suberecta Haw.

- - semiguttata Haw.

- tuberculata Haw.

- - major Nob. AL. echinata W.

- virens Haw.

(FROLIFERAE.)

- brevifolia $D C$. prolifera Schult.

- - fol. variegatis H. Dyck.

- depressa Haw.

- prolifera Haw. postgenita Schult.

- Serra $D C$.

\section{-}

-

(RHODACANTHAE.)

* Caule humili.

- glauca elatior Nob. glauca major Haw.

- - humilior Nob. glauca minor Haw.

- - spinosior Haw. Al. rhodacantha DC.

- - rubrocincta H. Dyck.

- lineata virescens Haw.

- - glaucescens Haw. 


$$
\begin{gathered}
\text { (11) } \\
\text { * Caule elato. }
\end{gathered}
$$

- crsia Nob.

- - tenuior Nob.

- gracilis Haw.

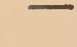

(SUBMACULATAE.)

- abyssinica Lam.

- vulgaris Lam. barbadensis Haw.

- - elongata Murr.

- - chinensis Haw.

- - elatior Nob.

$-$

(PANICULATAE.)

- albocincta Haw.

- paniculata Jacq. striata Haw.

$-$

'(SERRULATAE.)

- serrulata Haw.

- variegata Lin.

(MICROCANTHAE.)

- microcantlia Haw.

\footnotetext{
- microcantha Haw.
}

$$
\text { (PURPURASCENTES.) }
$$

- purpurascens Haw.

- succotrina Ait. sinuata Thunb. 


\section{(PICTAE.)}

- grandidentata Nob.

- latifolia Haw.

- pallescens Haw.

- picta DC. obscura Haw.

- - magnidens Haw.

- tenuifolia Lam.

- umbellata DC. saponaria Haw.

- fol. variegatis H. Dyck.

- distans Haw.

(MITRAEFORMES.)

* Spinis flavidis.

- flavispina Haw.

- mitræformis $W$.

- - elatior $W$.

- xanthacantha $W$.

\section{-}

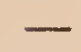

$$
\text { ** Spinis albidis. }
$$

- albispina Haw.

- Commelini W.

- spinulosa Nob.

(FRUTICULOSAE.)

- ciliaris Haw.

- tenuior Haw. 
(ARBORESCENTES.)

- arborescens $D C$.

- frutescens Nob.

- Salm Dyckiana Schult. PAcirI. Principis Haw.

\section{(PACHIDENDRA.)}

- africana Haw.

- - angustifolia Haw.

- ferox Mill.

- subferox Schult. pseudo-ferox Nob.
(RRIPIDODENDRA.)
- plicatilis Mill.
- major Nob.

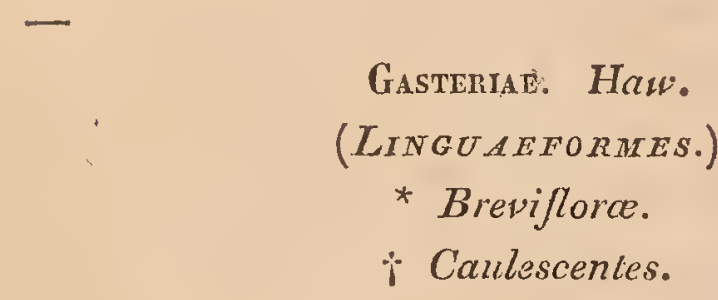

- Bowieana Schult. picta Haw.

- maculata $W$. obliqua Haw.

- - anguslior Nob.

- pulchra Jacq. obliqua DC.

- vittata Schult. fasciata How.

$-$

it Acaules.

- formosa Haw.

- Datifolia H. Dyck. 


\section{(14)}

- nigricans Haw. obliqua Jacq.

- crassifolia Nob. G. crassifolia Haw.

- marmorata Nob.

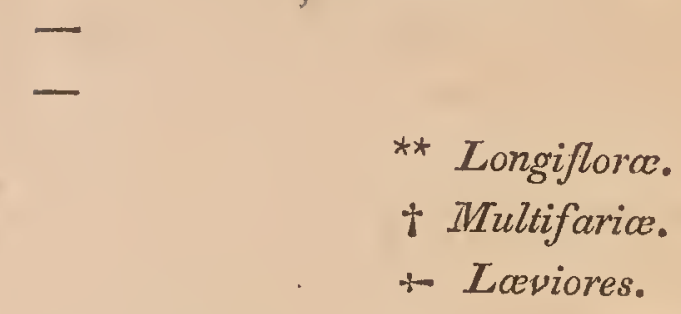

- acinacifolia Jacq.

- minor Haw.

- Bayfieldii H. Angl.

- candicans Haw.

- elongata Nob. trigona Haw.

- ensifolia Haw.

- glabra Haw.

- - minor H. Dyck.

- Iætepunctata Haw.

- linita Hazv.

- nitens Haw.

- fol. patentibus Haw.

- nitida parvipunctata Nob.

- - grandipunctata Nob.

- pluripunctata Haw.

- trigona Nob. obtusa Haw.

- undata Haw. 
- carinata Mill.

- pallidor Haw.

- decipiens Haw.

- pusilla Schult. parva Haw.

- repens Haw.

- strigata Haw.

- subcarinata Nob.

- - fol. variegatis Nob.

\section{t† Bifarioe.}

-Verrucosce.

- intermedia Haw.

- scaberrima Nob.

- subverrucosa grandipunctata Nob.

- parvipunictata Nob.

- verrucosa Ait. disticha Tunb.

- Latifolia Nob. carinata $D C$.

\section{$-$}

-

\footnotetext{
i- i- Laviores.

- angulata $W$.

- angustifolia Haw.

- - lævis Nob. G. lævis Haw.

- brachyphylla Nob. brevifolia Haw.

- conspurcata Nob.

- excavata $W$. latifolia Haw.
} 
- guttata latifolia Nob.

- - angustifolia Nob. subnigr. glabra Haw.

- lingua $W$. denticulata et disticha Haw.

- - latifolia Nob.

- mollis Haw.

- obtusifolia Nob.

- subnigricans Haw. pseudo-nigricans Nob.

- canaliculata Nob.

- sulcata Nob. angulata Haw.

Anacampseros Sims. - Rulingra Haw.

- arachnoides Sims.

- filamentosa Sims.

- lanceolata $D C$.

- polyphylla Haw.

- rubens $D C$.

- Telephiastrum $D C$.

- trigona $D C$.

$\overline{-}$

Anredera Juss.

- spicata Kunth. Basella vesicaria Lam.

Arenaria Lin.

- balearica Lin. 
- media Lin.

- peploides Lin.

Basella alba Lin.

- cordifolia Lin.

- Iucida Lin.

- rubra Lin.

Begonia acuminata Dry.

- argyrostigma Fisch.

- bulbillifera Schott. .

- dichotoma Jacq.

- dipetala Grah.

- disticha $L k$.

- Evansiana Bot. Mag. - discolor II. Kew.

- heracleifolia Otto.

- birtella $L R$.

- humilis Dry.

- insignis Grah. - incarnata Schott.

- longipes Bot. IMag.

- Martiana Lk. et Otto.

- monoptera Lk. et Otto.

- nitida Dry. - obliqua L'Her.

- - discolor.

- odorata W. 
- papillosa Grah.

- patula Fisch.

- platanifolia Schott.

- sanguinea Radd.

- semperflorens Lk. et Otto.

- Sellowii Otto.

- spathulata Horn. - pauciflora Bot. Reg.

- suaveolens Lodd.

- ulmifolia Humb.

- undulata Bot. Mag.

- vitifolia Schott. - elatior H. Berol.

BeLurem crassifolium.

Besleria melittifolia Lin.

- pulchella Bot. Mag.

- serrulata Jacq.

Bracirsstelma tuberosa R. Brow. - Stapela Meerb. 
Bryophyleuar calycinum Salisb.

Bulbine $W$. Anthericuni Haw.

- aloides Lir.

- annua $W$.

- ciliata $\mathscr{W}$.

- frutescens Lin.

- longiscapa Jacq.

- macrophylla Nob.

- narcissifolia Nob.

- pugioniformis Jacq.

- rostrata $\mathscr{W}$.

Bulliardia Vaillantii $D C$.

Cacalia Lin. Kueinia Haw.

- auteuphorbium Lin.

- articulata $W$. laciniata Jacq.

- canescens Nob. tomentosa Haw.

- cylindrica Lam. Oтronsa tenuissima Haw.

- ficoides Lin.

- Kleinia Lin. Kuetv. neriifolia Haw.

- longifolia Haw pugioniformis Nob.

- papillaris Lin.

- radicans Haw. carnosa Ait.

- repens Lin. 
CAKILE maritima Lam.

Calandrinia ciliata $D C$.

- compressa Schrad.

- discolor Schrad. speciosa Lehm.

- glauca Schrad.

- grandiflora Lindl.

- spectabilis Otto et Dietr.

Camaluuma R. Brow. Stapelia Roxb.

- crenata Vallich.

Ceneus DC. Cactus Lin.

(GLOBOSI.)

- denudatus Lk. et Otto.

- Eyriesii Turp.

- oxygonus Lk. et Otto.

-

(CEREASTRI.)

* Mriltangulares.

- multangularis $T V$.

- - albispinus H. Dyck.

- - prolifer H. Dyck. 
- senilis Haw. Bradypus Lehm.

- strigosus Hort. Angl.

-

** $6-12$ angrilares.

+ Lanuginosi.

- lanuginosus Lin. repandus Millo

- Royeni Lín. lanuginosus Mill.

- albispinus Nob.

故 Sublanuginosi.

- aureus Nob.

- - pallidior Nob.

- crenulatus Nob.

- - gracilior Nob.

- flavispinus $N o b$.

- Chexagonus Nob.

- fulvispinosus Haw. Royeni Mill?

- griseus Haw. Royeni $I V$.

- Haworthii Spreng, nobilis Haw.

- niger Nob.

- - gracilior Nob.

-
-

tit Glabri.

- candicans Hort. Angl.

- chiliensis Colla. 
- eburneus $N$. peruvianus $W$. coquimbanus Molli.

- - monstrosus Nob.

- Hystrix Nob.

- Olfersii Hort. Berol.

- peruvianus Taber. hexagonus $W$.

- - monstrosus DC. C. abnormis $W$.

- strictus $\mathscr{W}$.

- stictus

\section{itt Attenuati.}

- cærulescens Nob.

- eriophorus Hort. Beral.

- repandus Lin. Royeni DC. gracilis Mill.

- subrepandus Haw.

*** $3-5$ angulares.

- affinis Hort. Berol.

- glaucus Nob.

- jamacaru $\dot{D} C$.

- lrovirens Nob.

- lætus Kunth.

- oblusus Haw.

- pentagonus Lin. prismaticus et reptans. W.

- Pitahaja Jacq.

- sublanatus Nob.

- tetragonus Lin.

- - gracilior Nob. C. pentagonus $W$. 


\section{(23) \\ (Polylophi.)}

- cinerascens $D C$.

- - crassior DC.

- Deppei Hort. Berol.

- leptacanthus $D C$.

- crassior $D C$.

- propinquus $D C$.

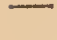

(SERPENTINI.)

* Globosi.

** Multangulares.

- flagelliformis Lin.

- - minor Hort. Dyck.

- leptophis $D C$.

- Martianus Zuccar.

- serpentinus Lag.

- Smithianus Hort. Angl.

-

ב

$* * * \cdot 5-6$ angulares.

- grandiflorus Lin.

- humilis $J C$. gracilis $N o b$.

- radicans $D C$. reptans $N o b$.

- spinulosus $D C$. 


$$
\begin{gathered}
(24) \\
* * * * 3-4 \text { cingulares. } \\
\text { i Speciosi. }
\end{gathered}
$$

- coccineus Nob. an C. bifrons Haw?

- speciosissimus Desf. speciosus Cav.

$$
\text { †† Tripteres. }
$$

- prismaticus Nob.

- triangularis Lin.

- major Nob. C. Napoleonis Hort.

- var. picta.

- tripteris Nob.

- triqueler Haw. prismaticus Desf.

- brevispinulus Nob.

- extensus Nob.

- setaceus Nob.

- - viridior Nob. C. coccineus $D C$.

\section{计 Fxtensi.}

$-$

\section{tht Squamulosi.}

- Myosurus Nob. tenuis Schott.

- - Lenuior Hort: Dyck.

- squamulosus $N o b$.

$$
-
$$

\footnotetext{
(A.LATI.)

* Corollis tubulosis.

- Ackermanni Haw.
} 


\section{( 25 )}

- Hoockeri Haw. C. Phyllanthus fl. majore DC.

- marginatus Hort. Dyck.

- p̈hyllanthoïdes $D C$. speciosus Bompl. alatus $W$.

- Curlisii.

- Quillardieri.

- - Jenkinsonii.

- - Vandesii.

- Phyllanthus Lin.

- truncatus Haw.

- - Altensteinii Hort. Berol.

alatus Swartz.

- - crassior H. Dyck.

- crispatus Haw.

- - latior H. Dyck.

- ramulosus Nob.

Ceroregra dichotoma Haw. aphylla $L k$. et Otto.

- stapelioides Haw.

Chevoronium frutescens Bieb. Salsola Lin.

- maritimum $W$. 
Cineraria Petasiles Bot. Mag.

- precox Cav.

- veslita Haw.

Crssus acida Lin.

- carnosa Lam.

- quadrangularis Lin.

- sicyoides Jacq.

Cilaytonia alcinoides Sims.

- perfoliata $W$.

- sibirica Lin.

- virginica $L i n$.

Cursis flava Jacq.

- rosea Lin.

Cotxlenón Lin. DC.

(F'oLILS OPROSITIS.)

- coruscans Haw.

- crassifolia Haw.

- orbiculata Lin.

- clata DC.

- - oblonga $D$. 
- obavata $D C$.

- - ramosa $D C$.

- papillaris Lin. decussata Bot. Mag.

- - tricuspidata Haw.

- ramosissima Nob.

- undulata Haw.

- ungulata Lam.

(Fotis alternis.)

* IMarcescentibus.

- canaliculata Haw.

- fasciculata Ait.

- spuria Lin.

- tuberculosa Lam.

\footnotetext{
-
}

\section{** Persistentibus.}

- clavifolia Haw.

- cristata Haw.

- hemispherica Lin.

- jasminiflora Nob.

- maculata Nob.

- triflora Nob. an Thumb? 


\section{(28)}

Crassula Lin.

$$
\text { (LATIFOLIAE) }
$$

- arborescens $\mathscr{W}$. C. Cotyledon

- lactea $A i t$.

- portulacea Lam.

- telephioides Haw.

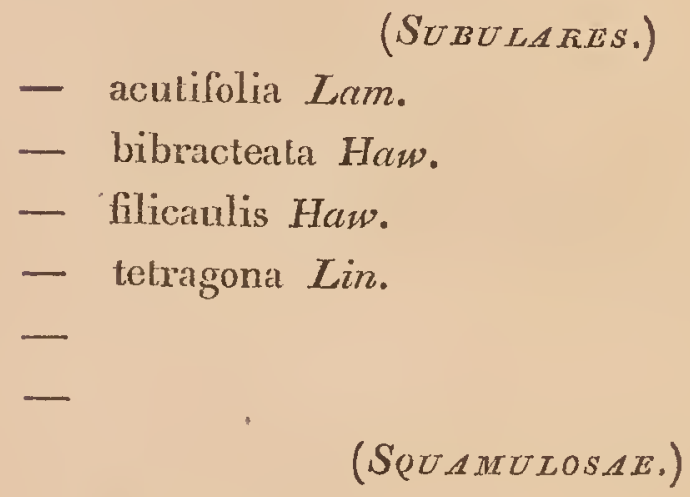

- scabra Lin.

- scabrella Haw.

- squamulosa $W$.

(CoLUMNARES.)

- columnaris Lin.

- ericoides Haw.

- Iycopodioicles Lam. C. imbricata Ail.

-

$$
\text { (PEREITATAZ.) }
$$

- marginalis Ait.

- perfossa Lam. 


$$
\begin{gathered}
(29) \\
(\text { PETIOLARES.) }
\end{gathered}
$$

- cordata Ait.

- spathulata Thunb.

(DELTOIDEAE.)

(TURGOSEAE.)

- ciliata Lin.

- conspicua Haw.

- corymbulosa Link.

- lingurefolia Haw.

- ramuliflora Link.



$-$

(ROSULARES.)

- orbicularis Lin.

- rosularis Haw.

$$
\text { (GLOMERATAR.) }
$$

(TILLAEOIDES.)

Cвiтrnnum maritimum Lin.

Gurtogine Haw. Crasstra Lin.

- dejecta DC. undata Haw.

- undulata Haw. 
Crmbidiun Swartz - Eprdendexi Lin.

- pendulum Sw. Epi. aloifolium Hort.

Dasystemion $D C$. Crassola $D e s f$.

Dramorpha Nutt. Sedum Mich.

Dianthus arboreus Lin.

Dischida bengalensis Collebr.

Dracaena brasiliensis Hort.

- cernua Jacq.

- Draco Lin.

- ensifolia Haw.

- ferrea $W$. terminalis Jacq.

- - var. picta.

- fragrans Haw. - ALkrRis fragrans Lin.

- ovata Gawl. - Aletris pumila Donn.

- reflexa Lam.

- salicifolia Hort. Berol.

- stricta Lin. - Cuariwoodia Sweet.

- tessellata $W$. marginata Lam.

- umbraculifera Jacq. 


\section{(31)}

Echeveria $D C .-$ Cotyledon $W$.

- cespitosa Haw. Cory. reflexa ' $W$.

- coccinea DC. Cotr. coccinea Cav.

- grandifolia Haw.

- racemosa Schlecht. livida Hort. Angl.

Echinocactus Link. et Otto. Cactus Lin.

$$
\text { * Aculeis rectis. }
$$

- aciculatus Nob.

- anfractuosus Mart. - (an E. crispatus $D C$.? )

- centeterius Lehm.

- corynodes Otto. E. rosaceus Hort. Berol.

- formosus Hort. Angl.

- gibbosus Haw.

- Gilliesii Hort Hamb.

- multiplex Hort. Berol.

- Ottonis Lehm.

- parvispineus Haw.

- polyacanthus Lk. et Otto.

- Scopa Lk. et Otto. 
** Aculeis recurvatis aut flexuosis.

\section{- armatus Nob.}

- cornigerus DC. (an latispinus Haw?)

- leucanthus Gillies. C. ambignus Hort.

- Linkii Lehm.

- muricatus Hort. Berol.

- phyllacanthus Hort.

- recurvus Haw. C. nobilis Lin.

- rhodacantus Nob.

- sessiliflorus Hort. Angl.

- tenuispinus Lk. et Otto.

EchITes suberecta Lin.

EPIDENDRUsi elongatum $S w$. 
Euphordia

\section{(CAULE CARNOSO.)}

- antiquorum Lin.

* Spinoso.

- arborescens Hort. Angl.

- canariensis Lin.

- cærulescens Haw.

- echinata Nob.

- erosa W. polygona Haw.

- heptagona Lin.

- Mystrix Jacq.

- lactea Haw.

- magnidens Haw.

- mammillaris Lin.

- neriifolia Lin.

- officinarum Lin.

- splendens Bot. Mag. - E. Millii H. Paris.

- trigona Haw.

- uncinata DC. Scolopendria Haw.

\section{** Inermi.}

- anacantha Ait. tridentata Lam.

- Caput Medusæ Lin.

- Clava Jacq.

- Commelini $D C$.

- globosa Haw. 


\section{$(34$ i}

- meloformis Ait.

- - pyriformis.

- tuberculata Jacq.

(CAUTE SUFFruticoso.)

- atropurpurea $D C$.

- balsamifera Ait.

- biglandulosa IIrw.

- Characias Lin.

- - fol. variegatis.

- colinifolia Lin.

- cyathaphora Jacq.

- dendroides Lin.

- genistoides Lin.

- loela Ait divaricata Jacq.

- lophogona $W$.

- mauritanica Lin.

- mellifera Ait.

- Paralias Lin.

- pendula Haw.

- piscaloria Ait.

- Pithyusa Lin.

- punicea $S w$.

- sanguinea.

- Tirucalli Lin.

- virgata Pers. 




- Chamaesyce Lin.

- hypericifolia Lin.

- Peplis Lin.

\footnotetext{
** Umbellis trifidis.

- exigua Lin.

- Peplus Lin.
}

*** Umbellis quadrifides.

- Apios Lin.

- Lathyris Lin.

\section{一 \\ **** Umbellis quinquefidis.}

- dulcis Lin.

- sylvatica Lin.

***** Umbellis multifidis.

- cyparissias Lin.

- Esula Lin.

- myrsinites Lin.

- palustris Lin.

Fagonia cretica Lin.

Fourcroya Zuccar.

- longœva Zuccar. 
Grsneria aggregata Ker.

- bulbosa Ker.

- latifolia Mart.

- prasinata Ker.

- rupestris Mart.

- rutila Lindl.

- spicata Lk. et Otto.

Grobulea Haw. - Crassura Lin.

(CULTRATAL.)

- cultrata Lin.

- radicans Haw.

\section{-}

(LINGUATAE.)

- capitata Nob.

- obvallata Lrom.

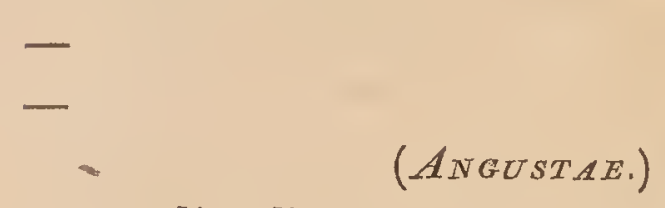

- nudicaulis Lin.

(LORATAE.)

- impressa Haw.

- paniculata Haw.

(SUBULATAE.)

- mesembryanthoides Haw. 
- mollis Lin.

- subincana decumbens Haw.

- - erecta Haw.

Gloxinra maculata $l^{\prime} H e r$.

- speciosa Ker.

- - alba.

- caulescens Bot. Reg.

$-$

Grammantmes $D C$. Vaunntmes Haw. Grassula Lin.

Hieracium fruticosum W. Simpria Monch.

Hora R. Brow. Schollia Jacq. Asclepias Lin.

- carnosa R. Brow.

- crassifolia Jacq.

- pallida Bot. Mag. acuta Haw.

Kalanchoe Adans. Verein Andr. Cotyledon Venlo

- acutiflora How.

- egyptiaca $D C$.

- ceratophylla How.

- crenata Haw.

- coccinea Hort. Berol. (an K. rolundifolia Haw?) 


\section{(38)}

- glauca Hort.

- laciniata $D C$.

- spathulata $D C$.

Lomatophyllum IV. - Phylloma Haw.

- borbonicum $W$. Aloe nargivalis $D C$.

- macrum Nob. Alos macra Haw.

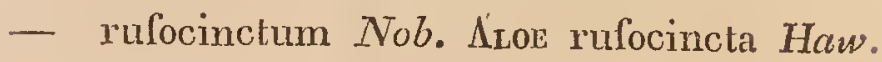

Manmilaria Haw. - Cactus lin.

(AXILIIS PROTIFERIS.)

* Hunteacanthe.

- angularis Hort. Berol.

- cirrhifera Mart.

- densa Lk. et Otto.

- Alavescens Haw.

- Hystrix Mart.

- Karwinskiana Zuccar.

- longimamma $D C$.

- macrothele Mart.

- magnimamma Haw.

- Parmentieri Hort. Berol.

- polyedra Mart.

- prolifera Haw.

- pycnacantha Mart.

- quadrispina Mart. 
- simplex Haw. C. mammillaris Lin.

- sphacellata Mart.

- subangularis $D C$.

- subcrocea $D C$.

- subpolyedra Hort. Dyck.

- tenuis $D C$.

- - media DC. M. Stella aurata Mart.

1 - tortolensis Hort. Berol.

$=$
$\overline{-}$
$=$
-
$\overline{-}$
$\overline{-}$

- bicolor Lehm.

- chrysacantha Hort. Berol.

- coronaria Haw. C. coronarius $W$.

- crinita $D C$.

- discolor Haw.

- eriacantha Otto. cylindrica Hort. Berol.

- fuscata Hort. Berol.

- geminispina Haw.

- glochidiata Marto crinita Var. DC.

- lanifera Haw.

- pusilla DC. stellaris W. Haw.

- - minor Hort. Berol.

- rhodantha Lk. et Otlo. 
- tentaculata Mart.

- vetula Mart.

\section{(MAMATILLIS PROLIFERIS.)}

- parvimamma Haw. microthele Spreng.

- vivipara Haw.

Melocactus C. Bauh. - Cagtus Lin.

- Beslerii Lk. et Otto.

- Communis Lk. et Otto. C. melocactus Lin.

- - atrosanguineus $L k$. et Otto.

- - viridis Lk. et Otto.

- ingens Korw.

- macracanthus Nob.

- meonacanthus Lk. et Otto.

- pyramidalis Nob.

- Salmianus Lk. et Otto.

- Sellowii $L k$. et Otto. 


\section{(41)}

Mresembryanthemum $\mathrm{Lin}$.

A. HOLIIS EPAPULOSIS.

I. SUBACAULIA.

(SPHAEROIDEA.)

- ficiforme Haw.

- minutum Haw.

- obcordellum Haw.

- perpusillum Haw.

(SUBQUADRIFOLIA.)

- fissum Haw.

- obtusum Haw.

- testiculare Jacq.

(MONILIFORMIA.)

(Rostrata.)

- bibracteatum Haw.

- bifidum Haw.

- denticulatum Haw.

- multipunctatum Nob.

- purpurascens Nob.

- quadrifidum Haw.

- ramulosum Haw.

- robustum Haw.

- rostratum Lin.

- - tuberculatum Haw. 


\section{(42)}

(ALOIDEA.)

- aloideum Haw.

- nobile Haw.

- ermininum Haw.

- felisum Lam.

- lupinum Haw.

- murinum Haw.

- musculinum Haw.

- mustellinum Nob.

- tigrinum Haw.

(RINGENTIA.)

* Sessiliflora.

-

** Scapigera.

- albidum Lin.

-- caninum $D C$.

- lybridum Haw.

- rulpinum Haw.

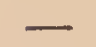

(DoLABRIFonMRA.)

* Acaulica.

- cerinans Haw.

- scapiger Haw.

** Subcaulescentia.

- dolabriforme Lin. 


$$
\begin{gathered}
(43) \\
\left(D_{\text {IFFORMLA. }}\right) \\
\text { * Ramosa. }
\end{gathered}
$$

- angustum pallidum Haw.

- - heterophyllum Haw.

- bidentalum Haw.

- - minus Haw.

- cruciatum Hav.

- difforme Haw.

- - brevicaule Haw.

- præpingue Haw.

- semicylindricum Haw.

** Acaulia.

- Salmii Haw.

- - elongatum Nob.

- - semicruciatum Nob.

(LINGUAEFORMIA.)

- adscendens Haw.

- cultratum læteviride Nob.

- - perviride Nob. M. mediun Haw.

- depressum Haw.

- - lividum Haw.

- fragrans Nob.

- grandiflorum Haw.

- latum Haw.

- - breve Haw.

- linguxforme Lin.

- - rufescens Haw. 
- - assurgens Haw.

- longrum Haw.

- - declive Haw. M. linguæforme DC.

- - angustius Haw.

- Iucidum Hewv.

- pustulatum How.

- scalpratum IIaw. obliquum IV.

- - minus.

- uncatum Nob.

- gibbosum Haw.

- luleo-viride Haw.

- perviride Haw.

(GIBBOSA.)

$-$

(CALAMIFORMIA.)

- calamiforme Lin.

(TERETIFOLIA.)

- cylindricum Haw.

\footnotetext{
(BELLIDIFLORA.)

- bellidiflorum simplex Lin.

- Wiplex Lin.

- subulatum Mill. bellidiflorum DC.

$-$

(ACUTA.)

- aculum IIav.

- diminutum Haw.
} 
- cauliculatum Haw.

\section{(MACRORHIZA.)}

II. CAULESCENTIA.

† TRIQUETRA.

(Cornicutata.)

* Diversifolia.

- diversifolium majus Haw.

- - atrovirens Haw.

- Lætevirens Nob. M. loreum Hà.

- congestum Nob. loreum congestum Haw.

- reptans Ait.

** Homophylla.

- corniculatum Lin.

- - isophyllum Haw. Br. corniculatunin DC.

- decipiens Haw.

- dubium Haw.

- procumbens Haw.

- purpureo-album Haw.

- stramineum $W$. tricolorum $H a w$.

- validum Haw.

\footnotetext{
(PUGIONIFORMLA.)

- brevicaule Haw.

- capitatum Haw.

- elongatum minus Haw.

- pugioniforme Lin.
} 
(46)

(SARMENTOSA.)

* Rigidiora.

- rigidicaule Haw.

- Schollii Nob. aduncum Jacq.

- simile Haw.

$$
\text { ** } \operatorname{Lax} \alpha \text {. }
$$

- geminiflorum Haw. geminatum Jacq.

- laxum Haw.

- sarmentosum Haw.

(REPTANTIA.)

- australe Ait. demissum $\mathscr{W}$.

- clavellatum Haw.

- crassifolium Lin.

\section{$-$}

$-$

\section{(ACINACIFORMIA.)}

* Australia.

- æquilaterale Haw.

- - decagynum DC. brevius Haw.

- chiliense Haw.

- glaucescens Haw.

- Rossi Haw.

- virescens Haw.

** Capensia.

- acinaciforme Lin.

- edule Lin. 


\section{(47)}

- rubrocinctum Haw.

- compressum Haw.

- tenerum Haw.

- snbalatum Haw.

(RUBRICAULIA.)

- filamentosum Lin.

- rubricaule Haw.

- - densius Haw.

- serrulatum Haw.

(HETEROPETALA.)

- glaucinum Haw.

- - crassius Hatv. M. tricolorum Jacq.

- heteropetalum Haw.

- inclaudens Haw.

- lacerum Haw. gladiatum Jacq. camnatum. Vent.

- mutabile $I$ aw. filamentosum DC. forficatum Jacq.

(BRACTEATA.)

- anceps Haw.

- compressum Haw.

- gracile Haw.

$-$

$-$

$-$

(VIRCATA.)

- cymbrefolium Haw.

- virgatum Haw. 


\section{(48) \\ ( VIRENTIA.)}

- virens Haw.

- - minus Nob.

(AUREA.)

- aurantium Haw.

- aureum Lin.

- glaucum Lin.

- - humilius Haw.

- $\quad\left(B_{L A N D A .)}\right.$

- blandum Haw.

- curviflorum Haw.

(AIOENA.)

- amoenum Nob.

- conspicuum Haw.

- formosum Haw.

- spectabile Haw.

(PALIIDIFLORA.)

- dilatatum Haw.

- incurvum Haw.

- - densius Haw.

- roseum $W$.

-

(FALCATA.)

- falcatum Lin.

- falciforme Haw.

- lunatum $W$. 
- maximum Haw.

- caulescens Mill.

- deltoides Lin.

- muricatum Haw.

(DELTOIDEA.)

(FORFICATA.)

- forficatum Lin.

(GEMINATA.)

- geminatum Haw. 
(MICROPHYLLA.)

- microphyllum Haw.

- mucronatum Hav.

- pulchellum Haw. canescens Haw.

- - revolutum Haw.

- pygmæum Haw.

(ROSTELLATA.)

- Rostellum Haw.

(VAGINATA.)

- acutangulam Haw.

- curtun Haw.

- - politum Haw.

- imbricatum minus Haw.

- - viride Haw.

- multiflorum majus Haw.

- - nitens Haw.

- patens Haw. M. patens ID.

- parviflorum Haw.

- rigidum Haw.

- - majus Harv.

- tenellum Haw.

- vaginatum asperum Haw.

- Chamatum Nob. H. hamation

$-$

$-$

(TUMIDULA.)

- foliosum Haw. 
- tumidulum Haw.

- - glaucescens Haw.

- umbellatum Lin.

\section{(CROCEA.)}

- croceum Jacq. insititium $W$.

- - purpureo-croceum Haw.

ก门 TERETIUSCULA.

( $V_{E R U C U L A T A .)}$

- veruculatum Lin.

(HAWORTIIANA.)

- corallinum Haw.

- Haworthii $W$.

- læve Haw.

- lepidum Haw.

- productum Haw.

- stipulaceum Lin.

\section{(SPINOSA.)}

- mucroniferum Haw.

- spinosum Lin.

(CXMBAEFORMIA.)

- cymbæforme Haw.

- molle majus Haw.

- - minus Nob. 


\section{(DEFOTIATA.)}

- defoliatum Haw. clavatum Jacq.

- fulvum Haw.

- noctiflorum phœniceum Haw.

- - elatum Haw.

- - stramineum Haw.

- luteum Haw.

$-$

- acuminatum Haw.

(SPLENDENTIA.)

- fastigiatum Haw.

- flexuosum Haw.

- splendens Lin.

- sulcatum Haw.

- umbelliflorum Jacq.

$-$

(JUNCEA.)

- granilicaule Haw.

- junceum Haw.

- micranthon Haw. parvillorum Jacq.

\section{-}

(TENUIFOLIA.)

- bicolorum Lin.

- coccineum $D C$.

- - acutius Haẁ.

- inæquale Haw.

- tenuifolium Lin.

- - erectius Haw. 
- variabile Haw.

$(A D U N C A$.

- aduncum Haw.

- curvifolium Haw. cerataphyllum $\mathscr{W}$.

- flexicanle Haw.

- flexifolium Haw.

- spiniforme Haw.

- - subaduncum Haw.

B. FOLIIS PAPULOSIS.

I. PAPILLOSA.

(SCABRIDA.)

* Staminibus expansis.

- emarginatum Lin.

- glomeratum Lin.

- polyanthon Haw.

- polyphyllum Haw.

- violaceum $D C$. puniceum Jacg.

** Staminibus collectis.

- deflexum Ait.

- retroflexum Haw. elegans Jacq.

- scabrum Lin.

- versicolór Haw.

-

(TRICHOTONLA)

- macrorhizum Haw. 


\section{(54)}

- subincanum Haw.

- tuberosum Lin.

\section{$-$}

(ASPERICAULIA.)

* Parviflora.

- brevifolium Haw. erigeriflorum Jacq.

- flavum Haw.

- obliquum Haw.

- parvifolium Haw.

- pulverulentum Haw.

$-$

** Grandiflora.

- micans Lin.

- speciosum Haw.

-

-

\section{(HISPIDA.)}

- attenuatum Haw.

- calycinum Haw.

- candens Haw.

- virescens Haw.

- floribundun Haw.

- hispidum Lin.

- striatum

- subcompressum Haw.

- Luberculatum DC. hispifolium Haw. 


\section{(55) \\ (BARBATA.)}

- birbatum Lin.

- bulbosum Haw.

- densum Haw.

- intonsum flo. rubro Harv.

- flo. albo Haw.

- stellatum DC. hirsulum How.

- stelligerum Haw.

$$
\text { (ECHINATA.) }
$$

- echinatun flo. albo Haw.

- flo. luteo Haw. M. selosum Mönch.

- strumosum majus Haw.

- - minus Haw.

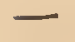

$$
\begin{aligned}
& \text { (SPINULIEERA.) } \\
& \text { * Foliis persistentibus. }
\end{aligned}
$$

- longispinulum Haw.

- nitidum Haw. brachiatum DC.

- spinuliferum Haw.

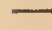

Foliis marcescentibus.

- grossum Haw.

- tenuiflorum Jacq.

- viridiflorum Ait.

\section{(CRASSLLINA.)}

- canaliculatum Haw. reflexum DC.

- crassulinum Haw.

- incomptum Haw. 
- salmoneum Haw.

(GENICULIFLORA.)
- geniculiflorum Lin.

(NODIFLORA.)

- nodiflorum Lin.

II. PLANIFOLIA.

(SCAPOSA.)

- tricolor $W$. pyropæum Haw.

(PLATYPHYLLA.)

- crystallinum Lin.

- pinnatifidum Lin fil.

(CORDIFOLIA.)

- cordifolium Lin fil.

-

(EXTANSA.)

- anatomicum Haw.

- crassicaule Haw.

- expansum Lin.

- tortuosum Lin.

- - rectum

- varians Haw.

-

(RELAXATA.)

- pallens Aiø. expansum $D C$. 
- relaxatum $I J$.

\section{(TRIPOLIA.)}

- Tripolium Lin.

\section{(HELIANTIIOIDEA.)}

- pomeridianium Lin.

(INCERTA SEDIS.)

- alsinoides Hornens.

Movmia fontana Lin.

Nirraria Schoeberi Lin.

Nolava paradoxa Lindl.

- prostrata Lin.

Optantia Tourn. Cactus Lin.

A. CAULE NON ARTICULATO.

I. CYLIN DRACEO.

(CYLINDRACEAE.)

- cylindrica Juss.

- decipiens $D C$.

- exuriata $D C$.

- imbricata Haw. (an 0. rosea DC.)

- Kleiniæ $D C$.

- leptocaulis $D C$.

- - lætevirens Hort. Dyck. 
- Pocppigii H. Berol.

- ramilifera Nol.

- Stapelia $D C$.

- tunicata Lehrn.

- brazilicnsis $\mathscr{W}$.

- (PARADOXAE. $)$

- - spinosior Hort. Dyck.

II. COMPRESSO.

(CRUCIFotames.)

- froxix $W$.

- rubescens Nor.

- spinosissima Mill.

-

B. CAULE ARTICUISATO.

\section{ARTICULIS SUBCYLINDRACEIS.}

$$
\text { (DIVARICATAE.) }
$$

- andicola Hort. Angl.

- articulala Hort. Berol.

- corrugata Hort. Angl.

- curassavica Lin.

- - minor Haw.

- - elongata Haw.

- Coliosa W. pusilla How.

- Pragilis Nutt.

- glomerala Haw. 


\section{(59)}

- horizontalis Gillies.

- longispina Haw.

- platyacantha Hort. Angl.

- pusilla Nob.

- sulphurea Gillies.

- tuberculata Hort. Angl.

\section{ARTICULIS COMPRESSIS.}

(ALBISPINOSAE.)

- albicans Nob.

- Amyclea Tenor. maxima Nob.

- candelabriformis Hort.

- cochinillifera Mill.

- decumana W. maxima Mill.

- decumbens Nob. repens Karw.

- dejecta Nob.

- elata Hort. Berol.

- glaucescens Hort. Berol.

T- leucacantha Hort. Berol.

- lencotriche $D C$.

- media Haw.

- megacantha Nob. Iongispina H. Berol.

- missouriensis DC. ferox Nutt. polyacantha Haw.

- sericea $N o b$.

- - longispina Nob.

- tomentosa Nob. 
- triacantha $\mathscr{W}$.

- Dillenii Haw.

$$
\text { (FLAVTSPINOSAE.) }
$$

- - minor Hort. Dyck.

- horrida Nob. humilis Haw.

- polyantla $D C$.

- pseudo-Tuna Nob.

- - spinosior Hort. Lyck.

- Tuna Mill.

- - levior Nob.

- - uno latere læviore Nob.

-
$\overline{-}$
-

(FULVISTINOSAE.)

- monacantha $W$.

- nigricans Haw. 


\section{(61)}

(SETACI:AE.)

- crassa Haw.

- Ficus indica Lin.

- - articulis brevioribus Hort. Dyck.

- intermedia Nob. vulgaris major H. Dyck.

- lanceolata Haw.

- microdasys Lehm.

- minor Nob. Op. pulvinata DC.

- parvula Nob.

- spinulifera Nob.

- stricta Haw. Op. inermis DC.

- - spinulescens Fort. Dyck.

- tuberculata $W$.

- vulgaris Mill. C. opuntia Lin. Op. nana $D C$.

Otrronna arborescens Lin.

- cacalioides Lin.

- crassifolia Mill.

- frutescens Lin.

Pelargonuur carnosum Ait.

- ceratophyllum $L^{\prime} H e r$.

- cotyledonis L'Her.

- dasycaulon Sims. 
- echinatum Curt.

- - flo. rubro.

- fulgidum Ait.

- gibbosum $W$.

- ignescens $S w$.

- - sterile. P. splendens $W$.

- lateripes L'Her.

- multiradiatum Wendl.

- peltatum Ait.

- - zonale.

- pulchellum Ait.

- sanguineum $W_{\text {endl. }}$

- scutatum $S w$.

- tetragonum L'Her.

- - fol. variegatis.

Pentrionum sedoides Lin.

Pereshia Plum. - Cactus Lin.

- aculeata Mill.

- - longispina Haw.

- - latifolia Hort. Dyck.

- grandifolia Haw. 


\section{(63)}

PIrer Lin.

(CAULE CARNOSO.)

* Foliis allernis.

- acuminatum Lirr.

- amplexifolium Link.

- brachypliyllum $T V$.

- cuneifolium Jacq.

- incanum Haw.

- maculosum Lin.

- magnoliæfolium Jacq.

- obtusifolium Lin. clusixefolium Jacq.

- pellucidum Lin.

- reniforme Poir.

- subrotundum Haw.

- - var. Hort. Dyck.

- talinifolium Humb. et Küuntr.

** Foliis verticillatis.

- aromaticum W. inzequalifolium Ruiz. et Pav.

- blandum Jacq.

- pereskiæfolium Jacq.

- polystachyon Ait. obtusifolium Jacq.

- verticillatum Lin. pulchellun How.

- rubellum Haw.

- septemnerve Vahl.

- rubricauIe Nees tricarinatum Hawo. 
Pistorina DC. - Cotyledon Lin.

Puectranthus fruticosus $L^{\prime} H e r$.

Plunieria alba $\operatorname{Lin}$.

- aurantiaca Don.

- bicolor Rz. et Pav.

Ponturacs oleracea Lin.

- pilosa Lin.

- sativa Haw.

Pontulacaria afra Jacq.

Prnnura Palinuri.

Reaumuna vermiculata Lin.

Riragodia hastata $R$, Brow. 


\section{(65)}

Ruipsalis Goort. - Cactus Lin.

- Cassytha Goert.

- - major Hort Dyck.

- - pendula Hort. Dyck.

- - pilosiuscula Hort. Dyck.

- fasciculata $W$. C. parasiticus $D C$.

- funalis Nob. grandiflora Haw.

- mesembryanthoides Haw.

- salicornioides Haw.

$\rightarrow$ - ramosior Nob.

Rochea DC. - Larochea et Katosaytures Haw.

- coccinea DC. Crassula Lin.

- falcata $D C$.

- - major Hort. Dyck.

- jasminea Haw.

- perfoliata Haw. Crassura Lin.

- odoratissima Haw.

- versicolor Haw.

Salicornia herbacea Lin.

- fruticosa Lin.

Sarsola Soda Lin.

- Tragus Lin. 
SANSEviERa carnea $W$.

- fulvocincta Haw.

- grandicuspis Haw.

- guineensis $W$.

- lætevirens Haw.

- pumila Haw. stenophylla Link.

- zeylanica $W$. Salma spicata Cav.

Sancostrama R. Brow. - Cynanchum Lin.

- viminale $R$. Brow.

Saxifraga Lin.

(ACAULIAE.)

- Aizoon Sternb.

- crustata Vest.

- erosa Pursh.

- hieracifolia Lapey.

- Iongifolia Lapey. S. Cotyledon Lin.

- pensylvanica 'Lin.

- pyramidalis Sternb.

- stellaris Lin.

- virginiensis Mich.

(SUBACAULIA.)
${ }_{* *}$ Foliis integris.

- crassifolia Lin.

- cordata W. S. cordifolia Haw. 
- cernua Lin.

- cuneifolia Lin.

- cuscutaformis Lodd.

- dentata Link.

- Geum Lin.

- hirsuta Lin.

- hybrida Vill.

- nivalis Lin.

- polita Link.

- rotundifolia Lin.

- sarmentosa Lin.

- serrata Otto.

- umbrosa Lin.

\section{$-$}

** Foliis divisis.

\section{- condensata Gmel.}

- cuspidata Schl.

- exarata Trans. Soc. Lin.

- granulata Lin.

- - flore pleno.

- hirta Haw.

- hypnoides Lin.

- incurvifolia Trans. Soc. Lin.

- intermedia Trusch.

- irrigua Marsch.

- moschata Sternb.

- muscoides Jacq.

- nervosa Lapey. 
- pedatifida Smith.

- pedemontana Allion.

- pentadactylis Lapey.

- Ponæe Sternb.

- pygmaea Trans. Soc. Lin.

- Sternbergii W. Sponheimica Gmel.

- tridactylites $\operatorname{Lin}$.

- trifurcata Sternb.

- villosa W. decipiens Ehrh.

(RAMULOSAE.)

- aizoides $W$.

- aspera Lin.

- bronchialis $W$.

- bryoides $W$.

- Burseriana Jacq.

- cresia Lirb.

- Hirculus Lin.

- oppositifolia Lin.

- planifolia Lapey.

- sedoides Lin.

- tenella Jacq. 
SEDur Lin.

$$
\begin{aligned}
& \text { (PIANIFOLIA. - AnaCampenos Haw.) } \\
& \text { * Floribus albis aut rubicundis. }
\end{aligned}
$$

- albicans Haw.

- Anacampseros Lin. A: sempervireus Haw.

- argutum Haw.

- Cepæa Lin.

- - galioides Albion.

- cruciatum Desf. monregalense Balb.

- Eversi Ledeb.

- hrmatodes Mill.

- ibericum Bieb.

- involucratum Bieb.

- latifolium Bert.

- lividum $\mathbb{W}$.

- multifforum Hort. Monast.

- oppositifolium Sims. A. ciliaris Haw.

- populifolium Lin.

- Rhodiola DC. Rhodiola rosea Lin.

- sempervivoides Fisch.

- spurium Bieb.

- stellatum Lin.

- Telephium Lin. A. vulgaris Haw.

- - purpureum Mich. A. purpurea Haw.

- telephioides Mich.

- ternatum Mich. S. portulacoides $W$.

- triphyllum Haw. 


\section{( 70$)$ \\ ** Floribus luteis.}

- aizoides Nob.

- Aizoon Lin.

- hybridum Lin.

-

(TERETIFOLIA.)

* Subulata. Floribus luteis.

- albescens Haw.

- altissimum $D C$. Semperv. altissimum Jacq.

- anopetalum $D C$.

- coerulescens Haw.

- crassicaule Hort. Berol. S. reflexum W.

- Forsterianum Smith. S. rupestre DC.

- hexapetalum Haw.

- minus Haw.

- ochroleucum Snitt.

- reflexum Lin. S. recurvatum W.

- cristatum Schrad.

- rostratum Tenor.

- rufescens Tenor.

- rupestre Lin.

- septangulare Haw.

- spirale Haw.

- virens Haw.

- virescens $W$.

- viridulum Haw. 
** Obtusizuscula.

+ Trloribus luteis.

- acre Lin.

- boloniense Lois.

- saxatile W. S. annuum Lin.

- sexangulare Lir.

to Fl. albis, nubris, aut caruleis.

- album Lin.

- anglicum Huds.

- atratum Lin.

- cæruleum Vahl.

- dasyphyllum Lin.

- glaucum WValdst. Kit.

- hispanicum Lin.

- neglectum 'Tenor.

- nudum Ait.

- pallidum Bieb.

- rubens DC. - Crassula Lin.

- turgidum Tenor.

- villosum Lin. 
Sempervivux $\operatorname{Lin}$.

$$
\begin{gathered}
\text { (Chronobia DC. }) \\
\text { * Fruticulosa. }
\end{gathered}
$$

- arboreum Lin.

- - foliis variegatis

- - foliis atropurpureis.

- aureun Smitt. S. calyciforme Haw.

- - spurium Haw.

- barbatum Smith. S. linieolare Haw.

- - hybridum Haw.

- canariense Lin.

- - subtabulæeforme Nob.

- cespitosum Smith. S. ciliatum Haw.

- glandulosum Ait.

- glutinosum Ait.

- Haworthii Hort. Angl.

- Snithii Sims.

- tabulæforme Haw.

- tortuosum Ait.

- urbicum Hom.

- villosum Haw.

- dichotomum $D C$.

- stellatum Smith. S. villosum Ait 
(JOVIBARBAE DC.)

- arachnoideum Lin.

- flagelliforme Fisch.

- Funkii Hoppe.

- globiferum Lin.

- hirtum Lin. S. soboliferum Bot. Mag.

- montanum Lin.

- tectorum Lin.

- Wulfenianum Hoppe.

\section{-}

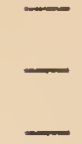

$($ Monantres Haw.)
- monanthes Ait. M. polyphylla Haw.

Senecio hæmotophyllus $W$.

Septas capensis Lin.

- - globifera $D C$.

$-$

Sestrium portulacastrum $J a c q$.

- revolutum $W$.

Sinningia Helleri $N, a b, E$. 


\section{(74)}

StaptLia Lizz.

A. COROLLIS CAMBHANULATIS.

* Decemfudis.

(HUERNIA R. Brow.)

- campanulata Mass.

- clavigera Jacq.

- crassa Donn.

- guttata Mass.

- lentiginosa R. Brow.

- reticulata Mrass.

- tubata $J a c q$.

- - duodecimfida Jacq.

- venusta Mass.

** Quinqueficlis.

(PoDAnthes et PIARANTIIUs Haw.)

- irrorala Mass.

- roriflua Jacq.

- verrucosa Mass.

\footnotetext{
B. GOROLLIS ROTATIS.

* Ramis glabris.

† Reptantibus prostratis.

(Obesia. - Duvalia et Carunculatiza. Haw.)

- articulata Mass.

- cespitosa Mass.

- concolor Nob.

- decora Mass.
} 
- geminala Mass.

- hirtella Jacq.

- marmmillaris $W$.

- maslodes Jacq.

- pedunculata IMass.

- radiata Jacq. S. Jacquiniana Schult.

- reclinata Mass.

- replicata Jacq. S. radiata Sims.

\section{$-$}

to Adscendentibus.

(ORBEA Haw.)

- acuminata Hort.

- anguinea Jacq.

- arida Hort.

- atropurpurea Nob.

- bidentata Hort.

- bisulca Donn.

- bufonia Jacq.

- clypeata Jacq.

- conspurcata W.

- Curtisii Haw. S. variegata Curt.

- gemmifera Hort.

- lepida Jacq. limosa Hort.

- maculosa Jacq.

- ligulis integris Hort. Dyck.

- marginata $W$.

- marmorata Jacq. 
- mixta Hort.

- normalis Jccq.

- - obscura H. Dyck.

- - orbiculo minimo H. Dych.

- - laciniis angustioribus H. Dyck.

- - corollis sæpe duplicis H. Dyck.

- obliqua $W^{r}$.

- orbicularis Andr.

- picta Donn.

- planifolia Jacq.

- var. H. Dyck.

- var. S. anguinea affuis H. Dyck.

- plicata Hort.

- quinquenervis Haw.

- rugosa Jacq.

- tridentata Hort.

- trisulca Jacq.

- valiegata Lin.

- - monstrosa.

- verlicillata Hort.

- Wendlandiana Schult. S. rugosa WVendl.

- Woodfordiana Hort. 
tit Erectis.

(TRIDENTEA et TroMLATRICHE Haiw.)

- gemmiflora Mass.

- glauca Jacq.

- hircosa Jacq.

- juvencula Jacq.

- mutabilis Jacq.

- - var. Jacq.

- revoluta Mass.

- vetula Mass.

\section{** Ramis pubescontibus.}

(Gonostemon el STAPELIA Haw.)

- ambigua Mass.

- - var. Jacq.

- Asterias Mass.

- comata Jacq.

- deflexa Jacq. reflexa Haw.

- - obscura Jacq. S. brevirostris $W$.

- dejecta Nob.

- divaricata Mass. pallida Wendl.

- grandiflora Mass.

- hirsuta Lin.

- - var. Jacq.

- - var. Hort. Dyck.

- lanifera Haw.

- paniculata $W$. 
- patula $W$. sororia Jacq.

- pulvinata Mass.

- rufescens Nob.

- sororia Mass.

- - Willdenowii. sororia var. Jacq.

- spectabilis Haw. grandiflora Sims.

- stellaris Jacq.

- uncinata W. sororia var. altera Jacq.

Talrsum Adans. - Pontulaca Lin.

- crassifolium $W$.

- patens $W$.

- teretifolium Pursh.

Tetragonia crystallina L'Her.

- decumbens Mill.

- echinata Ait.

- expansa Ait.

- fruticosa Lin. 
Tillaea muscosa Lin.

Tradescantia Crassula Link.

- crassifolia Cav.

- discolor L'Hert.

Truanthema monogyna Lin.

Unamicus $D C$. - Cotylanon Lin.

(ROSULARIA.)

(MUCIZONIA.)

$-$

(COTYLE.)

- erectus DC. Cotru. lutea Fuds.

- pendulinus DC. Corru. Umbilicus Lin.

$-$

(OROSTACHFS Fisch,

- malacophyllus DC. Cotruedon Pall.

- spinosus IJC. Corruedon Lin.

Urtica baccifera Lin.

- crassifolia $W$. 
VANLLLA aromatica $S w$.

-'planifolia Andr.

YucGa Lin.

(Fol. margine spinosis aut serrulatis.)

- acrotricha Hort. - an Schied?

- aloifolia Lin.

- - fol. variegatis.

- arcuata Haw.

- conspicua Haw.

- crenulata Haw.

- Draconis Lin.

- longifolia Karw.

- serratifolia Karw.

- tenuifolia Haw.

- filamentosa Lin.

(Fol. margine filiferis.)

- fol. variegatis.

- glaucescens Haw. angustifolia Hort.

- puberula How? arborea Hort.

- recurva Haw. 
(81)

(Fol. margine loevibus.)

- gloriosa Lin.

- obliqua Haw.

Zygophylu, album Lin.

- Fabago Lin.

- foetidum Schrad.

- Morgsana Lin.

- sessilifolium Lin. 


SMITHSONIAN INSTITUTION LIBRARIES

I IIIIII

(1) 39088017560079
0 